\title{
A time-frequency approach for generation of synthetic time-histories of earthquake signals
}

\author{
$\operatorname{Roshan}_{\text {kumar }^{1}} \cdot$ P. Sumathi ${ }^{2} \cdot$ Ashok Kumar $^{1}$
}

Received: 21 October 2014/Accepted: 23 April 2015/Published online: 8 May 2015

(C) Akadémiai Kiadó 2015

\begin{abstract}
Seismic signals are non-stationary in nature, generated by earthquake and some other sources like explosive, landslides etc. In this paper, pattern of time varying frequency components of real-time recorded data of seismic signal are investigated by computing the mean of Gabor transform. In most of the cases, it is not possible to use recorded data of strong ground motion at a given site. It is therefore essential to analyze synthetic timehistory which gives the prediction of strong ground motion in the regions where actual records can't be used. In this work, the problem has been shown in synthetic time-histories where time-frequency variation is not controlled and suggested Gabor transformation based approach for generation of site specific synthetic time-history having control on time-frequency variation. Synthetic time-histories have been generated at five different sites by computing the average of Gabor transform of eight records for different earthquakes at each site. It is also shown that shape of response spectrum calculated from the generated synthetic time-history at each site is quite comparable to shape of mean response spectrum of recorded time-histories. Synthetic time-histories of a realistic time-frequency variation will be very useful for the researchers who wish to perform the nonlinear analysis of structures for a particular site.
\end{abstract}

Keywords Gabor transform - Response spectra - Seismic $\cdot$ Time-history · Time-frequency

Roshan kumar

roshan.iit123@gmail.com

P. Sumathi

psumifee@iitr.ernet.in

Ashok Kumar

akmeqfe@iitr.ernet.in

1 Department of Earthquake Engineering, IIT Roorkee, Roorkee, India

2 Department of Electrical Engineering, IIT Roorkee, Roorkee, India 


\section{Introduction}

Seismic signal analysis based on frequency domain technique and time-frequency distributions (TFDs) have been carried out over the period of approximately five decades. The spectral analysis using Fourier transform measures the frequency components of signal (Huang and Pan 2011; Danisor et al. 2007), but fails to provide the temporal information of time varying signal (Qian and Dapang 1999; Lopez et al. 2000; Ferguson and Quinn 1994). For linear analysis of structures, temporal variation of frequency content may not be important but for non-linear analysis of structures time-frequency variation can make significant contributions. Therefore, time-frequency techniques have been applied in several applications of earthquake engineering such as preprocessing of seismic signals (Roshan et al. 2013; Lopez et al. 2000; Botero et al. 2012), and damage detection of structures in which frequency shifting pattern was observed (Roshan et al. 2014; Cano Saldana 2008).

The short time Fourier transform (STFT) is the simplest method of time-frequency representations (Qian and Dapang 1999; Lopez et al. 2000; Ferguson and Quinn 1994), which can be defined as Fourier transform of the product of the signal and shifted version of window function i.e. rectangular, Hanning, and Hamming window (Qian and Dapang 1999). The result of STFT suffers from the windowing effects due to the limitation given by uncertainty principle (Qian and Dapang 1999; Lopez et al. 2000; Ferguson and Quinn 1994; Nuruzzaman Abul et al. 2006; Khan et al. 2011) and another problem associated with STFT is possible amount of leakage (Douglas and Parks 1987; Stankovic 1994). For this reason, Gaussian window is introduced in place of Hanning or Hamming window and it turns out to be a Gabor transform as shown in (Zielinski 2001; Ding 2012). The application of Gaussian window in Gabor transform provides better resolution. Therefore, Gabor transform was employed to determine the instantaneous frequency and damage detection of Imperial County services building for Imperial Valley earthquake (Todorovska and Trifunac 2007). Further, Gabor transform is employed in this work to analyze the recorded data of earthquake signal and detailed study is performed for its use in synthetic time-history. The synthetic time-history provides the prediction of strong ground motion in the regions uncovered by instruments. Recorded earthquake time-histories are frequently employed for the design purposes. For example EI Centro, California, US 1940, this timehistory has been utilized all over the world for design purposes. However its special characteristics are not really applicable because researchers found some statistical fluctuations in intensity, duration and frequency contents in this record (Jennings et al. 1968). For this reason, researchers have generated ensembles of simulated time-history by various ways to fill the gap in the recorded data. Boore $(1983,2003)$ generated a simulated ground motion by computing Gaussian band-limited noise and employs random process. Further, Suarez and Montejo (2005), generated artificial earthquake signal using the wavelet transform. The artificial earthquake signal was generated for five stations by considering a single time-history for each site. However, the synthetic signal generated from a recorded time-history, cannot be a reliable approach. Research is continued in the direction of simulating a synthetic ground motion and developed a model by (Rezaeian and Der Kiureghian 2010). This model shows acceleration time-history as the response of a linear filter with time varying parameters to white-noise excitation that is normalized by its standard deviation and is multiplied by a time modulating function to obtain the acceleration time-history. This simulation model has been extended to generate synthetic orthogonal horizontal components of ground motion in (Rezaeian and Luco 2012). 
Recently, Joshi et al. (2014) employed a semi-empirical technique for simulation of strong ground motion and compared the simulated data in terms of root mean square error (RMSE).

In this paper, synthetic time-history is generated by computing the inverse of mean of the Gabor transform of recorded time-histories at one station and its time-frequency variation is compared to the synthetic time-history without considering the time-frequency variation (Kumar 2002, 2004). Synthetic time-histories are generated for five stations by computing the mean of Gabor transform of eight records from different earthquakes at a station and finally it is transformed back to reconstruct the signal in time-domain by computing the inverse of Gabor transform. In this work, it is also shown that shape of response spectrum calculated from synthetic time-histories at each station matches well with mean response spectrum of recorded time-histories.

\section{Overview of fast Fourier transform and time-frequency distributions}

\subsection{Fast Fourier transform}

In the fast Fourier transform (FFT) algorithm, the discrete Fourier transform (DFT) operation can be decomposed into DFTs of shorter lengths to reduce the number of computational complexity. FFT provides the exact information about the frequency component of a signal, but this approach does not provide the information about the time at which the particular frequency exists in the signal (Huang and Pan 2011; Qian and Dapang 1999; Ferguson and Quinn 1994). Hence, the TFD are introduced for better spectral analysis and detection of the seismic signal. Joint time-frequency analysis offers the information of the signal to be analyzed in both the time and frequency domains simultaneously.

\subsection{Gabor transform}

The Gabor transform of signal is defined as,

$$
G T(t, f)=\int_{-\infty}^{+\infty} x(\tau) e^{\frac{-(\tau-t)^{2}}{2}} e^{-j 2 \pi f \tau} d \tau
$$

where $x(\tau)$ is the signal to be analyzed, $e^{\frac{-(\tau-t)^{2}}{2}}$ is Gaussian window and $t$ is the time shift of the window. While $\Delta t$ and $\Delta f$ are time and frequency standard deviations that must satisfy the uncertainty relation (Qian and Dapang 1999; Boashash 1996, 2003),

$$
\Delta t \times \Delta f=1 / 4 \pi
$$

Gaussian signal is the only signal which holds this equality, and for all other signals $\Delta t \times \Delta f>1 / 4 \pi$. Gabor transform is widely used due to its less spectral leakage. The advantage of Gaussian function over STFT is that the product of the standard deviation in time-domain and the standard deviation in the frequency domain assumes minimum theoretical value. This implies that a small time bandwidth product extracts more information of a signal. Since the Gaussian signal possess good energy concentration than the rectangular function in the frequency domain (Zielinski 2001; Boashash 1996) the frequency resolution of the Gabor transform is better than STFT. 
The original signal can be reconstructed into time-domain by computing the inverse of Gabor coefficients. The mathematical representation for inverse Gabor transform is expressed as

$$
x(\tau)=\int_{-\infty}^{+\infty} \int_{-\infty}^{+\infty} G T(t, f) e^{j 2 \pi f \tau} d \tau d f
$$

\section{Synthetic time-history without control on time-frequency content}

For the purpose of analysis of earthquake resistant structures, realistic strong ground motion is required. In most of the cases, it is not possible to have strong motion records at a given site. Even if such recordings are available, there is no basis to expect that a future earthquake might generate same or similar ground motion. It is, therefore, essential that for seismic time-history analysis of a structure, synthetic time-histories need to be generated for specific sites. One such work carried out by (Kumar 2002, 2004) is briefly described below.

Algorithm begins with the first iteration and it approximates that the target velocity response spectrum which is considered as Fourier magnitude spectrum of the history. To generate the frequency domain representation of the Fourier magnitude, random phase method is used and its time-domain representation of the signal is computed by applying the inverse of Fourier transform. This time-domain signal is multiply by an envelope function to obtain the non-stationary accelerogram. The response spectrum of this nonstationary signal is computed and compared at every bin of frequency. Further, correction factor is computed and modify the real and imaginary parts of Fourier transform of signal. Above iteration is continued till a satisfactory matching is obtained. It is apparent that above algorithm does not have control on time-frequency variation of generated timehistory.

\section{Methodology}

In this section, a new approach has been developed to generate the synthetic time-histories of earthquake signals using a time-frequency method i.e. Gabor transform. The synthetic time-histories are to be generated by computing the mean of the Gabor transform. The reason of considering the mean of Gabor transform is to smooth out the unwanted characteristics of signals.

The steps involved in the proposed work are as follows:

1. The eight earthquake time-histories are acquired for each of the five stations of Japan, namely Honbetsukai, Atsutoko, Ochiishi, Nemuro and Nosappu.

2. Each data set is normalized by dividing the peak ground acceleration.

3. Gabor transform of each time-history is computed with the help of Eq. (1).

4. Determine the mean of the Gabor transform (MGT) for each of the station using Eq. (4). 


$$
\operatorname{MGT}(t, f)=\frac{1}{n} \sum_{i=1}^{n} G T_{i}
$$

where $n$ is the number of time-histories.

5. The MGT of each station is converted into time-domain by computing the inverse of MGT by Eq. (3). The reconstructed signal is considered as a synthetic time- histories.

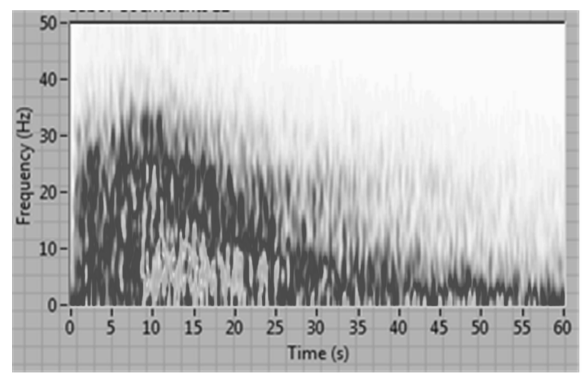

(a)

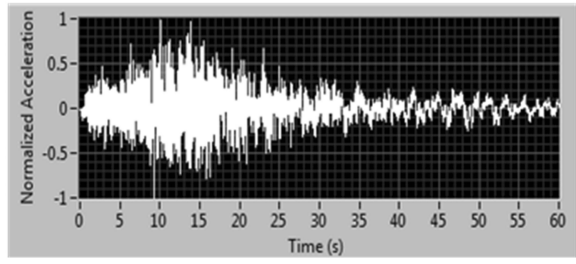

(b)

Fig. 1 a Mean of Gabor transform of eight time-histories recorded at Honbetsukai b Time-domain representation of mean of Gabor transform of Honbetsukai

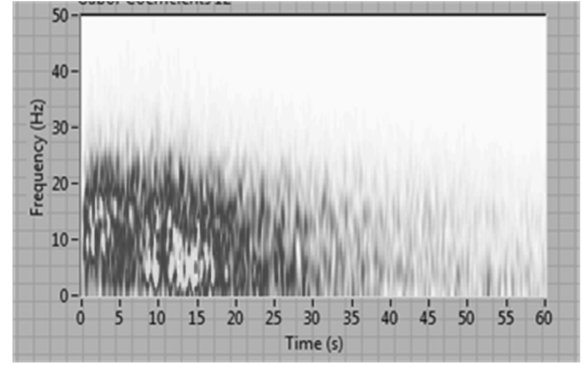

(a)

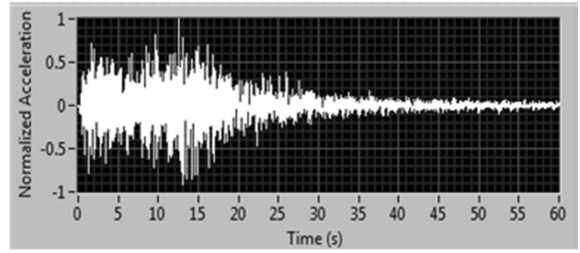

(b)

Fig. 2 a Mean of Gabor transform of eight time-histories recorded at Atsutoko b Time-domain representation of mean of Gabor transform of Atsutoko

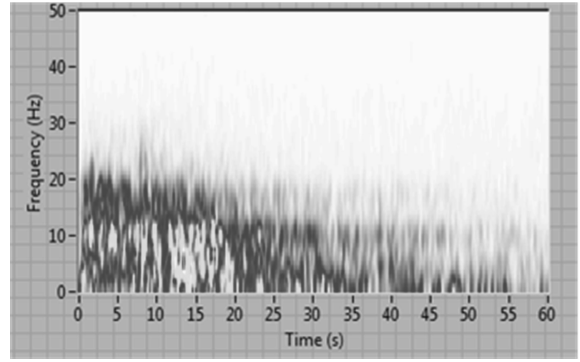

(a)

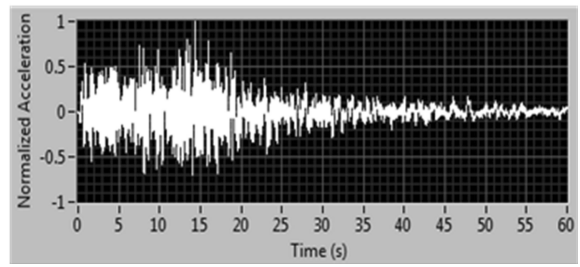

(b)

Fig. 3 a Mean of Gabor transform of eight time-histories recorded at Ochiishi b Time-domain representation of mean of Gabor transform of Ochiishi 


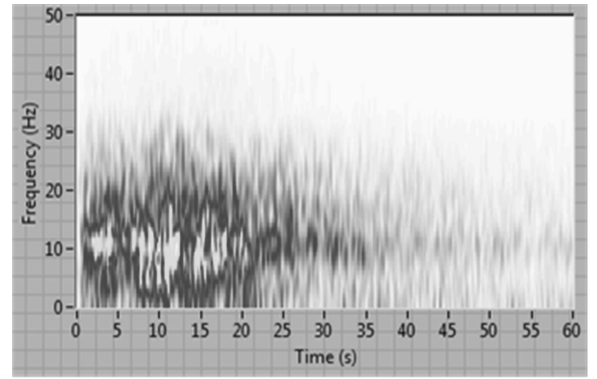

(a)

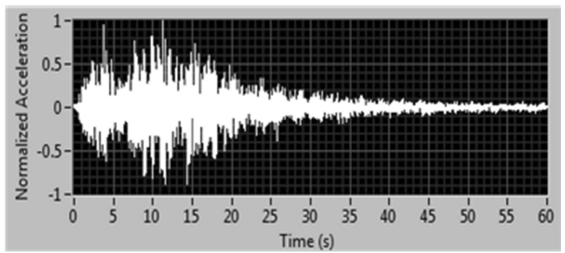

(b)

Fig. 4 a Mean of Gabor transform of eight time-histories recorded at Nemuro b Time-domain representation of mean of Gabor transform of Nemuro

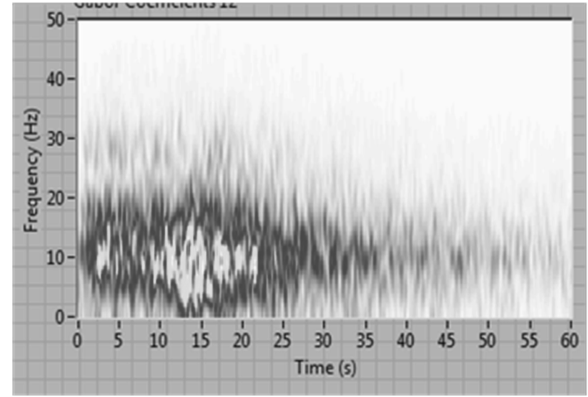

(a)

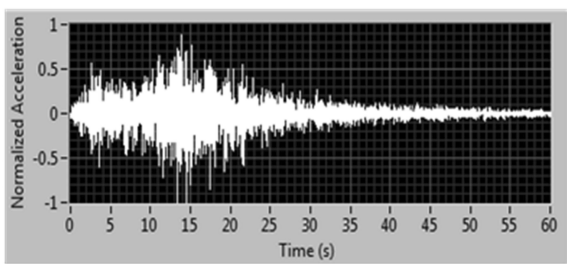

(b)

Fig. 5 a Mean of Gabor transform of eight time-histories recorded at Nosappu b Time-domain representation of mean of Gabor transform of Nosappu

\section{Results and discussion}

Seismic signals recorded by strong ground motion accelerographs from few stations of K.NET Japan, are considered for the analysis and the results are presented in this section. The joint time-frequency characteristic of seismic signal demonstrates interesting features from the seismological point of view.

In this work, the time-histories of ground acceleration recorded at Honbetsukai, Atsutoko, Ochiishi, Nemuro and Nosappu in Japan for eight different earthquakes at each of these sites (http://www.kyoshin.bosai.go.jp/), are considered for the analysis. These earthquakes magnitude are ranging from 5.0 to 6.0, and stations are at epicentral distance of $100 \mathrm{~km}$ or less. The length of the earthquake data employed for analysis is 6000 samples starting with P-onset with sampling rate of $100 \mathrm{~Hz}$ frequency i.e. $60 \mathrm{~s}$ record after P-onset. This time-history is divided by its peak ground acceleration to obtain the normalized timehistory. This normalized time-history is employed for further analysis.

Analysis of seismic data has been performed with Gabor transform. Gabor transform employs Gaussian window which possesses good energy concentration in time-domain as well as in frequency domain. The analysis using GT was carried out on five different Japan stations namely Honbetsukai, Atsutoko, Ochiishi, Nemuro and Nosappu. From each of these stations, eight time-histories were captured and Gabor transform was computed for 


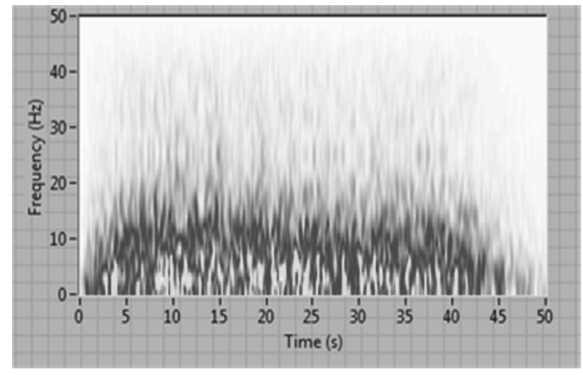

(a)

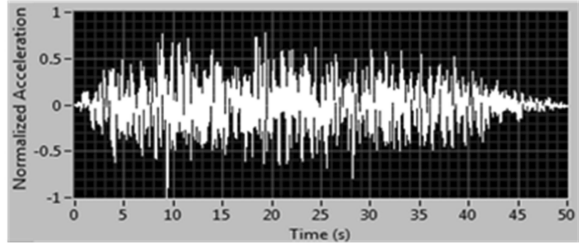

(b)

Fig. 6 a Mean of Gabor transform of the synthetic signal generated from the conventional method b Timedomain representation of mean of Gabor transform of synthetic signal generated from the conventional method

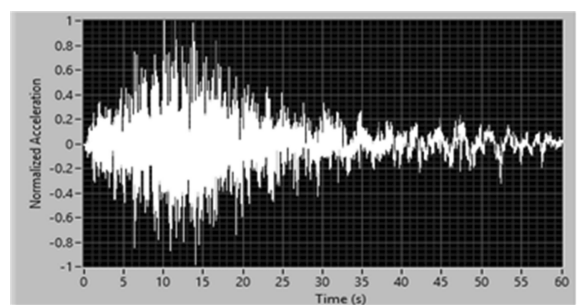

(a)

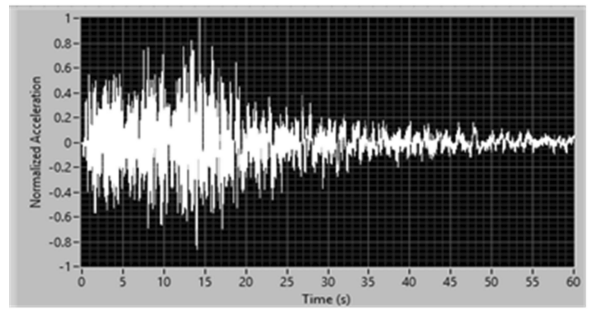

(c)

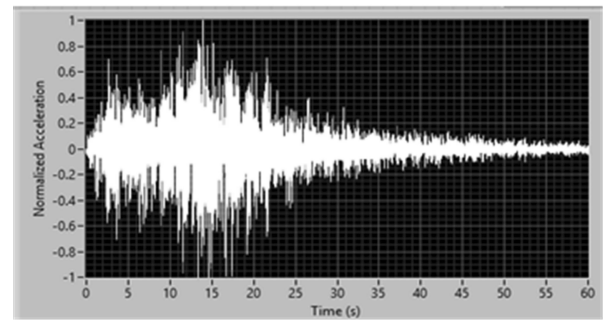

(e)

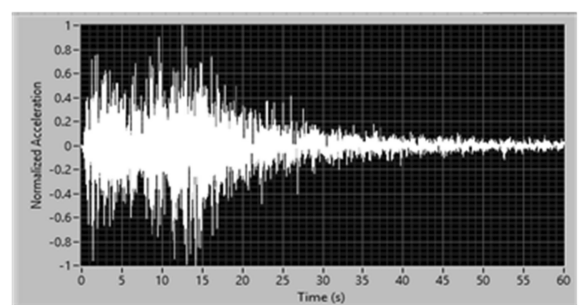

(b)

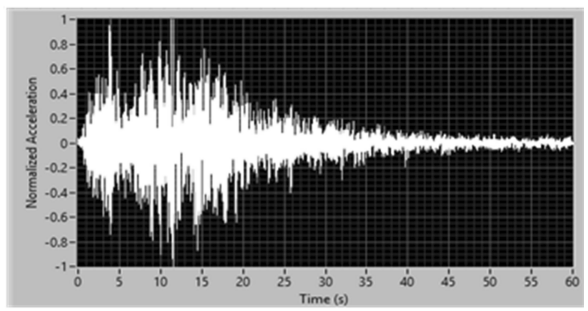

(d)

Fig. 7 Comparison of normalized time-history, mean + standard deviation and mean-standard deviation time-history for stations a Honbetsukai b Atsutoko c Ochiishi d Nemuro e Nosappu. (Color figure online)

each time-history. The mean of Gabor transform for each stations are shown in Figs. 1a, 2a, 3a, 4a, and 5a and its corresponding time-domain representations have been determined by computing the inverse of mean of Gabor transform are shown in Figs. 1b, 2b, 3b, 4b, and $5 \mathrm{~b}$. This time-domain representations are considered as a synthetic time-history. 


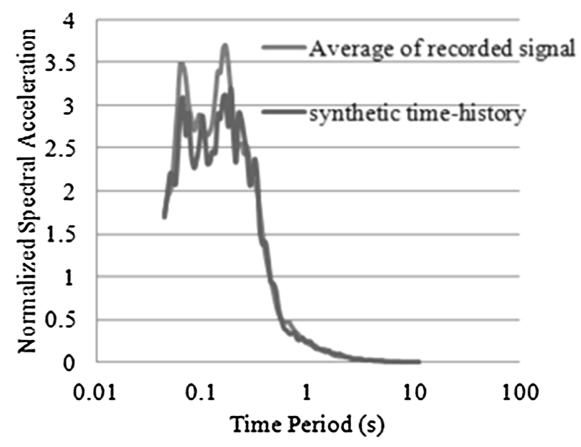

(a)

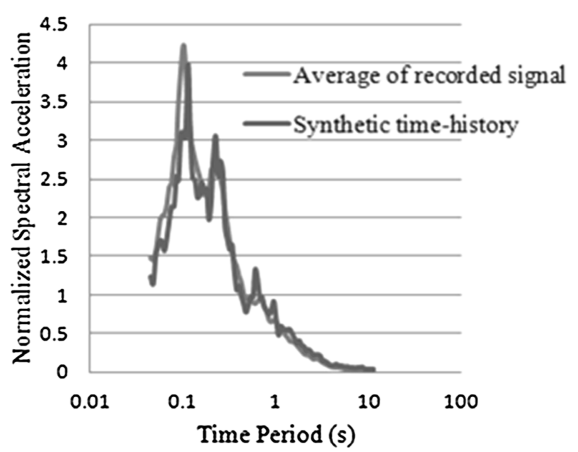

(c)

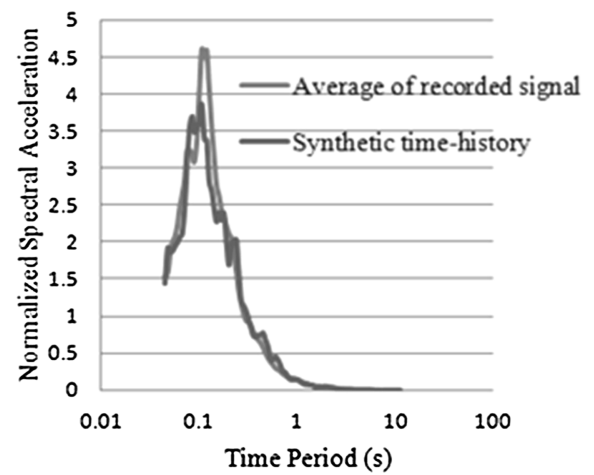

(e)

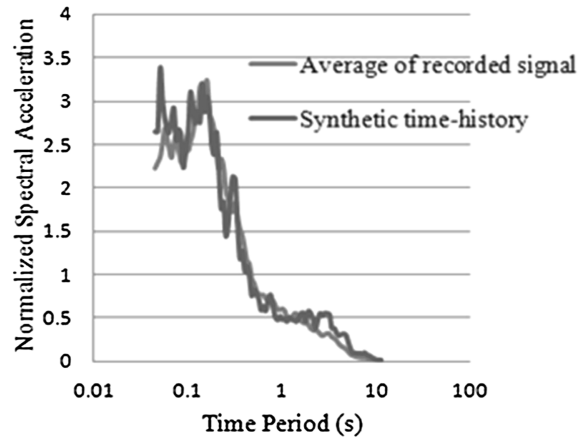

(b)

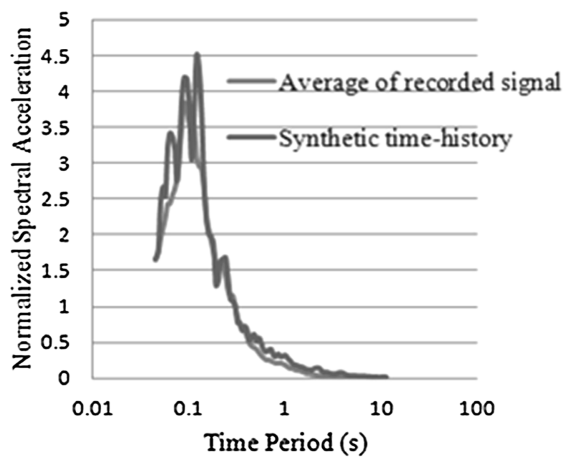

(d)

Fig. 8 Comparison of response spectra between average of recorded signal and synthetic time-history for stations a Honbetsukai b Atsutoko c Ochiishi d Nemuro e Nosappu. (Color figure online)

To compare the performance, eight synthetic time-histories without control on timefrequency have been generated and Gabor transform for each time-history was computed. The mean of Gabor transform of these synthetic time-histories is as shown in Fig. 6a and its corresponding time-domain representation can be seen in Fig. 6b. From the mean of Gabor transform of real-time recorded data, it is clear that the pattern of energy concentration over the time-frequency plane is quite similar to the realistic signals. However, 


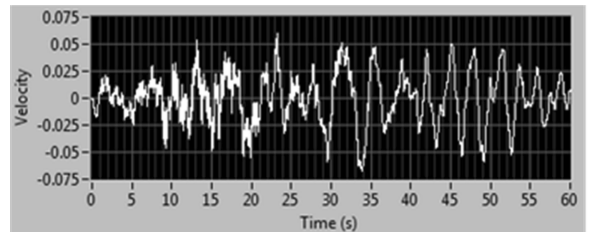

(a)

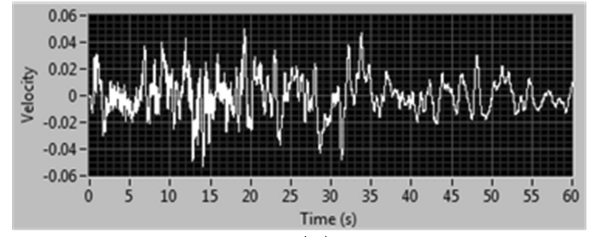

(c)

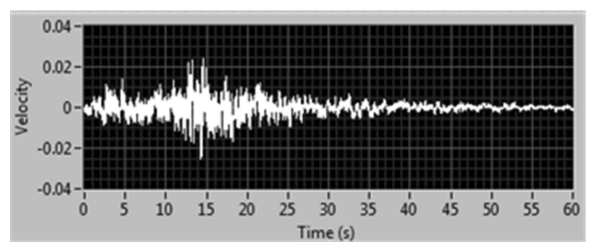

(e)

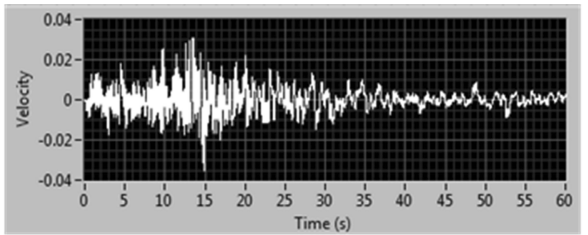

(b)

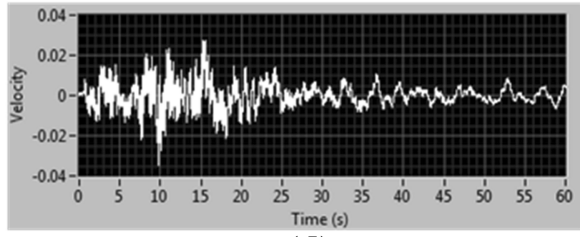

(d)

Fig. 9 Velocity time-history of normalized acceleration for stations a Honbetsukai b Atsutoko c Ochiishi d Nemuro e Nosappu

pattern of the time-frequency components of synthetic seismic signals generated from basic Fourier transformation approach (Kumar 2002, 2004), as can be seen from Fig. 6a, is not similar to the mean of Gabor transform of real data and it does not represent the actual scenario of energy distribution of real earthquake signals. Moreover, the analyses are continued and mean \pm standard deviation time-histories are also computed. In order to show the effectiveness of the proposed method, normalized acceleration time-history and mean \pm standard deviation time-history for each station, have also been calculated and presented on the same axis as can be seen in Fig. 7. In Fig. 7, white, green and red color represents the normalized acceleration time-history, mean \pm standard deviation and meanstandard deviation time-history respectively, and it is apparent that standard deviation is very low which shows the strength of the proposed method.

To ensure that the generated time-histories with our method should be similar overall effect on linear structures. The response spectrum computed from synthetic time-history generated by time-frequency approach, and compared it with average shape of spectra of eight earthquakes. In this work, response spectra for five sites have been studied and it's comparison can be seen in Fig. 8. It can be seen that average response spectrum of recorded histories matches quite well with response spectrum of synthetic time-history for all five sites. Further, velocity and displacement time-histories have also been calculated from synthetic accelerograms, which look quite reasonable and it can be seen in Figs. 9 and 10 respectively. 


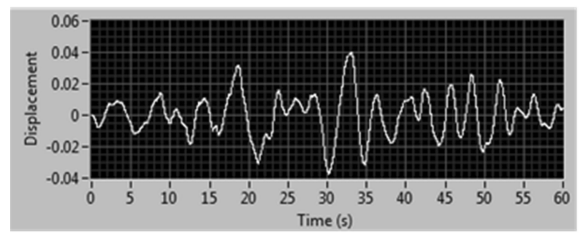

(a)

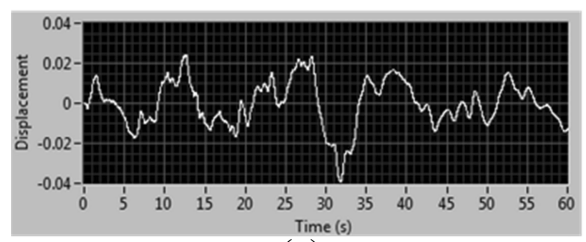

(c)

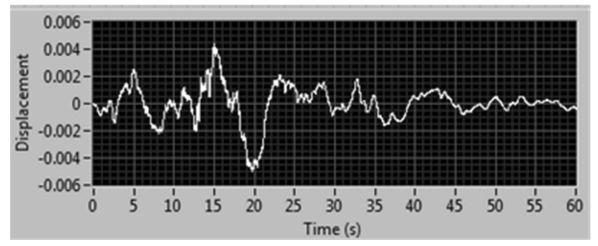

(e)

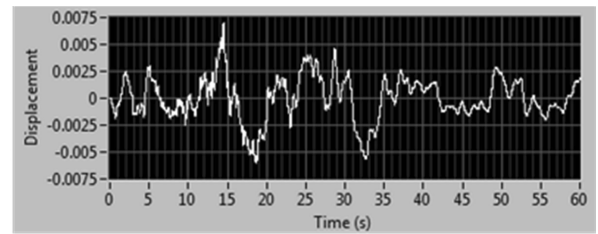

(b)

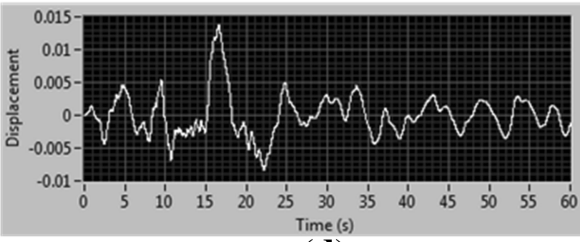

(d)

Fig. 10 Displacement time-history of normalized acceleration for stations a Honbetsukai b Atsutoko c Ochiishi d Nemuro e Nosappu

\section{Conclusion}

In this work, a method has been proposed to generate the synthetic time-history having control on time-frequency content for sites where few earthquake records are available. Further, response of the mean of Gabor transform of synthetic time-history, were compared with the mean of Gabor transform of synthetic signal generated from the basic Fourier transform approach (Kumar 2002, 2004) and observed that distribution of frequency components over the time-frequency plane do not match to realistic pattern of signals in case of synthetic signal generated by conventional methods. Therefore, it is suggested that that synthetic time-histories generated without control on time-frequency content may be applied cautiously particularly for nonlinear analysis. To investigate the performance of synthetic time-history, response spectra was computed and compared it with response spectra of average of recorded signals, which proves that the pattern of response spectra are quite similar to the average of recorded signals.

\section{References}

Boashash B (1996) Time frequency signal analysis: Past, present and future trends. Signal Processing Research Centre. Control Dyn Syst 78:1-69

Boashash B (2003) Time-frequency analysis and signal processing-a comprehensive reference. Elsevier, Oxford

Boore DM (1983) Stochastic simulation of high-frequency ground motions based on seismological models of the radiated spectra. Bull Seismol Soc Am 73(6):1865-1894 
Boore DM (2003) Simulation of ground motion using the stochastic method. Pure Appl Geophys 160(2003):635-676

Botero FMU, Huerta Lopez CI, Caro-Cortes JA, Martínez-Cruzado JA, Suarez-Colche LE (2012) Joint time-frequency analysis of seismic records. 15th World Conference on Earthquake Engineering

Cano Saldana L (2008) On time-frequency analysis for structural damage detection. Ph.D Thesis, University of Puerto Rico Mayagüez Campus

Danisor A, Izet-Unsalan KO, Unsalan D (2007) A new approach for the detection and processing of seismic signals. IEEE 3rd International conference on recent advances in space technologies, pp 698-703

Ding J-J (2012) Time-frequency analysis tutorial Gabor feature and its application. Tutorial, R99942057, pp $1-25$

Douglas LJ, Parks TW (1987) Time-frequency window leakage in the short time Fourier transform. Circuit Syst Signal Process 6(3):2222-2225

Ferguson BG, Quinn BG (1994) Application of the short-time Fourier transform and the Wigner-Ville distribution to the acoustic localization of aircraft. J Acoust Soc Am 96(821):821-827

Huang M, Pan BY (2011) Application of signal analysis in seismic wave processing. IEEE International Conference on e-Commerce and e-Government, pp 1-4

Jennings PC, Housner GW, Tsai NC (1968) Simulated earthqauke motions for design purposes. California Institute of Technology: Earthquake Engineering Research laboratory A-1:145-160

Joshi A, Sandeep, Kamal (2014) Modeling of strong motion generation areas of the 2011 Tohoku, Japan earthquake using modified semi-empirical technique. Nat Hazards 71:587-609

Khan NA, Noman Jafri M, Saad AQ (2011) Improved resolution short time Fourier transform. In the procedding of IEEE 7th international conference on emerging technologies, pp 1-3

Kumar A (2002) Generation of spectrum compatible time-history. Proceedings of 12th Symposium on Earthquake Engineering

Kumar A (2004) Software for generation of spectrum compatible time-history. 13th World Conference on Earthquake Engineering

Lopez CIH, Yong JS, Powers EJ, Roesset JM (2000) Time-frequency analysis of earthquake records. 12th World Conference on Earthquake Engineering, WCEE

Nuruzzaman Abul, Ozdal Boyraz, and Bahram Jalali (2006) Time-stretched short-time Fourier transform. IEEE Transactions on Instrumentation and Measurement, 55

Qian S, Dapang C (1999) Joint time-frequency analysis: IEEE Signal Processing Magazine, 16:52-57

Rezaeian S, Der Kiureghian A (2010) Simulation of synthetic ground motions for specified earthquake and site characteristics. Earthq Eng Struct Dyn 39(10):1155-1180

Rezaeian S, Luco N (2012) Example applications of a stochastic ground motion simulation methodology in structural engineering. 15th World Conference on Earthquake Engineering, WCEE

Roshan K, Sumathi P, Ashok K (2013) De-noising of seismic signals based on Gabor transform. IEEE International Conference on Advances in Computing, Communications and Informatics, 1996-2001

Roshan K, Sumathi P, Ashok K (2014) Frequency Shifting Analysis of Seismic Signals Using GaborWigner transform. Earthq Eng Eng Vib (accepted)

Stankovic L (1994) A method for time-frequency analysis. IEEE Trans Signal Process 42(1):225-229

Suarez LE, Montejo LA (2005) Generation of artificial earthquakes via the wavelet transform. Int J Solids Struct 42(21):5905-5919

Todorovska M, Trifunac M (2007) Earthquake damage detection in the imperial county services building: the data and time-frequency analysis. Soil Dyn Earthq Eng 27(6):564-576

www.kyoshin.bosai.go.jp/

Zielinski TP (2001) Joint time-frequency resolution of signal analysis using Gabor transform. IEEE Trans Power Deliv 50(5):1436-1444 